\title{
Forschungsprojekt „Neuartige Plattenwärmeübertrager"
}

FKZ 1711203/BMBF-AiF

\author{
Josef Schmadl, Jens-Uwe Gerking, Maik Haß, Ingrid Schult, Joachim Schult
}

\section{Einleitung}

Plattenwärmeübertrager bieten gegenüber Rohrbündelapparaten technische Vorteile durch effektivere Wärmeübertragung und damit verbunden Kostenvorteile. In den letzten Jahrzehnten haben gedichtete Plattenwärmeübertrager erhebliche Marktanteile gewonnen. Bedingt durch ihre Bauart aus geprägten Wärmetauscherplatten und Abdichtungen mittels Elastomerdichtungen sind sie in ihren Anwendungsmöglichkeiten jedoch auf einen Temperaturbereich zwischen $-10{ }^{\circ} \mathrm{C}$ bis $160{ }^{\circ} \mathrm{C}$ begrenzt.

Durch die Entwicklung eines neuartigen Fertigungsverfahrens zum automatischen Verschweißen von Einzelplatten zu Plattenpaketen werden die Produkträume statt mit Dichtungen durch das Verschweißen der Platten gegeneinander abgedichtet. Dieser kompakte Apparat ohne jegliche Dichtungen kann bei niedrigeren und höheren Temperaturen und hohen Drücken eingesetzt werden. Damit eröffnen sich neue Einsatzmöglichkeiten in der Kälteindustrie, der chemischen Industrie, in der Prozesstechnologie - hierbei insbesondere im zweiphasigen Nassdampfbereich - und im Bereich der Nahrungsmittelproduktion.

Erkenntnisse über Wärmeübergang und Strömungsbedingungen der geprägten Plattenwärmeübertrager in den nun möglichen Temperatur- und Druckbereichen liegen für die technische Wärmeübertragung flüssig/flüssig nur begrenzt und für Verdampfung und Kondensation im zweiphasigen Nassdampfbereich überhaupt nicht vor, siehe [1].

Dieses angewandte Forschungsvorhaben, ein Kooperationsprojekt der TFH Wildau und der Apparatebaufirma Caloperm (KMU), Niederlehme, will einen Beitrag zur Entwicklung vollverschweißter Plattenwärmeübertrager mit neuartigen, bisher nicht eingesetzten, kreisförmigen Platten (Abb. 1 und 2) mit folgenden Projektzielen leisten:

a. Experimentelle Leistungsmessungen an Plattenwärmeübertrager-Prototypen in halbtechnischem Maßstab im TVT-Technikum der TFH Wildau zwecks Optimierung der Konstruktion und Fertigung durch die Firma Caloperm im Hinblick auf Maximierung der erreichbaren Wärmestromdichte in $\mathrm{W} / \mathrm{m}^{2}$ und Minimierung des Druckverlustes,

b. Untersuchung der Verwendbarkeit dieses Apparatetyps für Siede- und Kondensationsanwendungen und

c. Erarbeitung adäquater Berechnungsgrundlagen für diesen neuen Apparatetyp.

\section{Teil A: Literaturauswahl zum einphasigen, konvektiven Wärmeübergang in Platten- wärmeübertragern}

Der im letzten Jahrzehnt zunehmende Einsatz der Plattenwärmeübertrager führte $\mathrm{zu}$ einer Vielzahl von Untersuchungen zur Beschreibung der charakteristischen Eigenschaften der Wärmeübertragung in Platten. Allerdings werden hauptsächlich die gebräuchlichen Rechteck-Platten (siehe Abb. 3) betrachtet. Nur wenige Abhandlungen beziehen sich auf andere Geometrien, z. B. runde Platten. Untersuchungen zum Einsatz von Wärmeübertrager für Medien mit Phasenveränderungen sind gegenwärtig

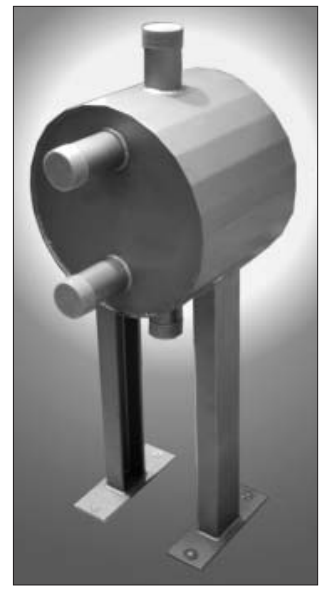

Abb. 1: Typischer Testapparat dieser Arbeit kaum in der Literatur vorhanden. Aufgrund der vielen Einflussgrößen sind die Methoden der Auslegung komplexer.

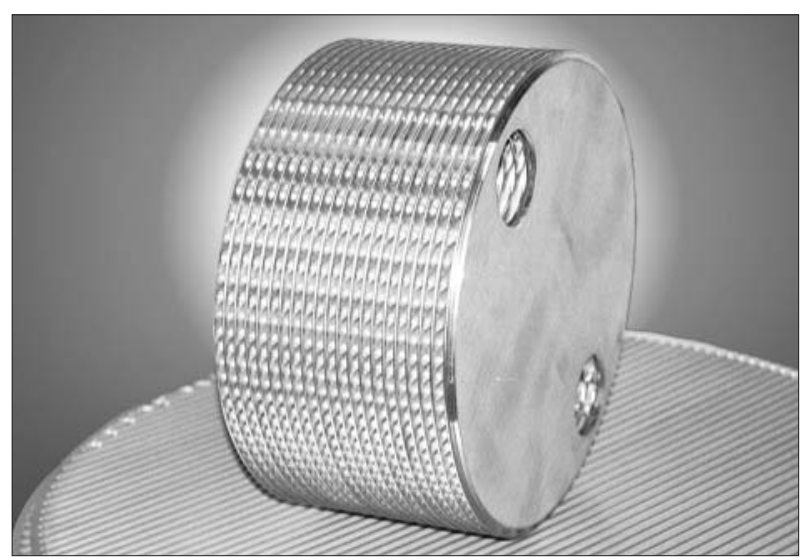

Abb. 2: Typisches Plattenpaket der getesteten Apparate

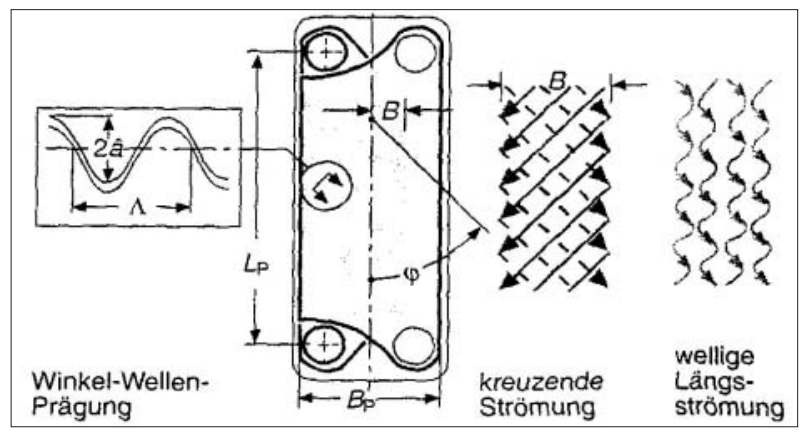

Abb. 3: Rechteck-Platte in Winkel-Wellen-Prägung. Winkel $\varphi$, Amplitude $\hat{a}$ und Wellenlänge $\Lambda$. 
Im Folgenden wird eine Zusammenfassung der wesentlichen Literaturzitate zum konvektiven Wärmeübergang in Plattenwärmeübertragern gegeben und im Hinblick auf die Verwendbarkeit in dieser Arbeit untersucht. Einen guten Überblick der für die Auslegung von Plattenwärmeübertragern in der Literatur benutzten Gleichungen für verschiedene Plattengeometrien und Bautypen sowohl für Einphasen-Medien als auch für Phasengemische wird dabei von Z. H. Ayub [2] gegeben. Er zeigt auf, dass Berechnungsgleichungen für die Nußelt-Zahl und Druckverluste bei 2-Phasengemischen noch ungenügend bestätigt werden, da nur einige wenige anfängliche Untersuchungen vorliegen.

\section{Experimentelle Arbeiten aus der Literatur}

Die meisten Untersuchungen zum Wärmeübergang und Druckverlust von Plattenwärmeübertragern liegen aus integralen Messungen über den Gesamtapparat vor. In diesen experimentellen Arbeiten werden im Wesentlichen die Einflüsse der Geometrieparameter auf den Wärmeübergang in Abhängigkeit von dimensionslosen Kennzahlen (Re, Pr) untersucht. Eine Messmethode ist die Visualisierung der Strömungsvorgänge hinsichtlich des Geschwindigkeitsprofils, der Ansammlungen und der Verweilzeiten im Apparat, um den konvektiven Wärmeübergang zu quantifizieren und örtlich relevante Abhängigkeiten festzustellen. Einige experimentelle Arbeiten werden im Folgenden zitiert.

M. Gaiser: „Strömungs- und Transportvorgänge in gewellten Strukturen" [3]

Diese Arbeit beinhaltet eine umfassende Analyse des Einflusses unterschiedlicher Geometrieparameter auf den Wärme- und Stoffübergang und dem zugeordneten Druckverlust in sinusförmig gewellten Strukturen. Die örtlichen Strömungsübergänge, die das Misch- und Verweilzeitverhalten des Fluids in solchen Strukturen wesentlich beeinflussen, werden in visuellen Untersuchungen sichtbar gemacht. Die experimentellen Untersuchungen erfolgten sowohl im Windkanal als auch im Wasserkanal. Aus den Strömungsuntersuchungen lässt sich ableiten, dass die gewellte Struktur eine rautenförmige Grundstruktur besitzt. Die unterschiedlichen Strömungsformen können auf Vorgänge innerhalb einer einzelnen Raute zurückgeführt werden. Der Autor weist zwei Grundströmungsarten - Längs- und Talströmung - in Abhängigkeit von den Geometrieparametern Wellenlänge/ Amplitude und Prägewinkel nach, die im technisch interessanten Bereich als Mischform aus der Überlagerung beider Strömungsarten vorliegt. Plattenstrukturen, die einen hohen Wärme- und Stoffübergang aufweisen, zeigen auch hohe Druckverluste. Allein durch die Variation der Geometrieparameter lässt sich bei gleicher Re-Zahl der Druckverlust um den Faktor 2,5 variieren.

In seinen Messungen zeigt sich bei gewellten Strukturen kein sprunghafter Umschlag von laminar zu turbulent wie bei der Rohrströmung, sondern es ergibt sich ein allmählicher Übergang. Mit wachsendem Anströmwinkel nimmt der Widerstandsbeiwert stark ab. Kleine Anströmwinkel bewirken viele Umlenkungen mit jeweiligem Wechsel der Strömungsrichtung, wodurch eine höhere Reibung, aber auch ein höherer Wärmeübergang erzielt wird.
R. Hoffmann, W. Krug und M. Arras: „Auswertung experimenteller Untersuchungen des Wärmeübertragungsprozesses im Plattenwärmeübertrager" [4]

Die Autoren beschreiben den Versuchsaufbau für die Untersuchung des thermischen Verhaltens von Plattenwärmeübertragern bei turbulenten Strömungsverhältnissen. Anders als in unserem Versuchsaufbau (3 Wärmeübertrager für Wärmezufuhr, -abfuhr und den eigentlichen Testapparat) werden hier alle Teilaufgaben in den 3 Abteilungen eines Plattenwärmeübertragers konventioneller Bauart realisiert. Verzichtet wird von den Autoren auf die Messung des Druckverlustes des Testsegments.

An Hand gewonnener Messdaten vergleichen die Verfasser zwei nicht näher erklärte Auswertungsmethoden auf eine an Hand der Veröffentlichung nicht nachvollziehbare Art und Weise. Als Ergebnis wird schließlich eine Wärmeübergangsbeziehung angegeben mit einem Gültigkeitsbereich von $1800<\mathrm{Re}<7400$ und $1,9<\mathrm{y} \operatorname{Pr}<23$ : Nu = 0,229 $\operatorname{Re}^{\star *} 0,68 \operatorname{Pr}^{* *} 0,43$ (pr/prw) ${ }^{\star *} 0,25$ Da nur Messungen mit Wasser beschrieben werden, erscheint die Grenze $\operatorname{Pr}<23$ äußerst zweifelhaft.

Der zweite Teil der Arbeit beschreibt den Fall veränderlicher Eintrittstemperaturen in den Plattenwärmeaustauscher. Die Auswertung der instationär vorgenommenen Messungen erfolgt nach einer nicht näher erläuterten nummerischen Methode.

W. W. Focke, J. Zachariades und I. Olivier: "The effect of the corrugation inclination angle on the thermohydraulic performance of plate heat exchangers" [5]

Die Autoren untersuchen den Einfluss des Prägewinkels auf den Wärmeaustausch und den Druckverlust. Die Testplatten haben eine Größe von 100 x 440 mm, die Prägewinkel variieren zwischen 0 und $90^{\circ}(0,30$, $45,60,72,80$ und $\left.90^{\circ}\right)$. Angewendet wird eine elektrochemische Massenübertragungsanalogie, die Aufschluss über die Strömungsverhältnisse gibt. In die Testplatte mit gewelltem Strömungsprofil wurden Elektroden eingelassen, die die Messung der aktuellen Ionenkonzentration gestatten. Mit ansteigendem Prägewinkel nimmt der Druckverlust zu; bei ca. $80^{\circ}$ wird der höchste Druckverlust erreicht. Bei Winkeln zwischen 80 und $90^{\circ}$ nimmt der Druckverlust ab. Die Messungen zeigen eine optimale Wärmeübertragung bei einem Winkel von $80^{\circ}$. Die Korrelationsgleichungen werden für verschiedene Turbulenzbereiche und Prägewinkel angegeben, die insbesondere für unsere Arbeit wichtig sind. Z. B. für einen Prägewinkel von $72^{\circ}$ für turbulente Strömung ergibt sich der Reibungsfaktor $\mathrm{f}$ als $\mathrm{f}=132^{\star} \mathrm{Re}^{-0,296}$. Die Arbeit zeigt auf, dass ein vielfaches Zick-Zack-Muster der Prägewinkel keine Verbesserung der Wärmeübertragung bringt. Bei der Sichtbarmachung der Fließbewegung zeigt sich, dass bei Winkeln bis $80^{\circ}$ die Flüssigkeit größtenteils längs der Furchen jeder Platte fließt und diese Strömung eine sekundäre Wirbelbewegung durch die querlaufenden Strömungen längs der entgegengesetzten Wand erfährt. Die sekundäre Wirbelbewegung bestimmt den Wärmeübertragungsprozess. 
M. Ciofalo, I. Di Piazza, Stasiek, J.A. : "Investigation of flow and heat transfer in corrugated-undulated plate heat exchangers (Untersuchung der Strömung und des Wärmeübergangs in Plattenwärmeübertragern mit gewellten Platten)“ [6]

Die Verfasser untersuchten experimentell Wärmeübergang und Druckverlust in Plattenwärmeübertragern mit gewellten Platten mit Luft als Versuchsmedium. Die Kühlung der erwärmten Luft erfolgte über Wasser. Die Messungen der Wandtemperaturen erfolgten nur auf der Luftseite mit auf der Oberfläche der Wärmetauscherfläche angebrachten Flüssigkristallelementen, die temperaturempfindlich farblich reagieren. Die Auswertung erfolgte über ein Bildverarbeitungssystem.

Des Weiteren führen die Autoren eine nummerische 3D-Untersuchung der Strömungsverhältnisse der Platten mit einem auf dem Finite-Volumen-Verfahren basierendem Computercode durch und vergleichen die Ergebnisse mit den experimentellen Resultaten.

Zwei aufeinanderfolgende gewellte Platten der vorgestellten Apparate unterscheiden sich jeweils durch ihre Wellenlänge und die Wellenhöhe. Die von uns eingesetzten Platten haben hingegen stets die gleichen diesbezüglichen Parameter. Somit erscheint eine Übertragung der Ergebnisse auf die von uns verwendeten Platten nicht relevant. Die experimentelle Bestimmung der Wandtemperaturen ist höchst interessant, aber nur sehr aufwändig zu realisieren.

\section{Theoretische Arbeiten}

Bei der Auslegung eines Plattenwärmeübertragers kommt es darauf an, dass die gewählte Plattenstruktur zu einem möglichst hohen Wärmeübergang bei akzeptablem Druckverlust führt. In allen Anwendungsfällen haben regelmäßige, symmetrische gewellte Plattenstrukturen in gekreuzter Anordnung die größte Bedeutung erlangt. Dabei gilt es die Strömungsvorgänge zu erfassen, die die Verweilzeit des Mediums im Apparat und die Austauschvorgänge bestimmen. Bisherige Untersuchungen beziehen sich fast ausschließlich auf integrale Werte, da Untersuchungen über die örtlichen Beziehungen von Druckverlust und Wärmeaustausch sehr aufwändig sind. Deshalb wird versucht, die Auswirkungen der Plattenstrukturen auf diese Parameter festzustellen, um die für den Prozess optimale Plattengeometrie auszuwählen. Grundsätzliche Literaturzitate zu Plattenwärmeübertragern werden im Folgenden aufgeführt.

P. Loose: „Teillastverhalten von Plattenwärmeübertragern" [7]

Der Autor zeigt in diesem Beitrag Berechnungsmöglichkeiten für das Teillastverhalten von Plattenwärmeübertragern am Beispiel der üblichen Warmwasserversorgung durch Fernwärme. Er bezieht dabei den Teillastfall auf den Auslegungsfall bei deutlich größeren Volumenströmen. Es sollte überprüft werden, ob dieser Weg nicht umgekehrt für die Auswertung unserer Messungen - die aufgrund der geringen übertragbaren Wärmemengen häufig im Teillastfall vorgenommen werden - anwendbar ist und somit die Abschätzung relevanter Wärmeübergangsparameter für den gewünschten Auslegungsfall erlaubt.
Diese allgemein für den Plattenwärmeübertrager geltende Arbeit geht nicht auf spezielle Typen (von Platten, Plattenwärmeübertragern) ein.

Ch. Ranganayakulu, K. N. Seetharamu: „The combined effects of inlet fluid flow and temperature nonuniformity in cross flow plate-fin compact heat exchanger using finite element method (Kombinierter Effekt ungleichförmiger Strömungs- und Temperaturverteilung im Fluideintrittsquerschnitt eines berippten KompaktPlattenwärmeübertragers mittels FEM)“ [8]

Die Autoren dieser Arbeit untersuchen den Einfluss der Strömungs- und Temperaturverteilung im Eintrittsquerschnitt eines Plattenwärmeübertragers auf den übertragenen Wärmestrom. Die Lösung der aufgestellten Modellgleichungen - für den im Kreuzstrom betriebenen Apparat - erfolgt mittels einer Finite-Element-Methode. Als Ergebnis dieser Arbeit wird deutlich, dass unterschiedliche Strömungs- und Temperaturverteilungen sehr erheblich auf die Leistung von Plattenwärmeübertragern einwirken können.

Da die praktische Anwendung dieser Arbeit die Kenntnis der Temperatur- und Strömungsprofile voraussetzt - die nur sehr aufwändig ermittelt werden können - scheint sie für die Verwendung im Rahmen unseres Projekts nicht sinnvoll.

\section{A. Klein: „Wärmeübertrager“ [9]}

Der Autor gibt einen allgemeinen Überblick über den gegenwärtigen Stand der Wärmeübertrager und geht dabei auch auf Platten- und Kompaktwärmeübertrager und Verdampfer ein. Unter anderem werden die Produktspektren von mehreren Herstellern vollverschweißter Apparate kurz umrissen und auch ein Anbieter vollverschweißter Apparate mit runden Platten angeführt. Hierbei wird der Arbeitsbereich mit $\mathrm{T}=-200 \mathrm{bis}+600^{\circ} \mathrm{C}$ bei Betriebsdrücken bis 100 bar angegeben. Die Platten haben einen Durchmesser von 190-998 mm und Übertragungsflächen bis $480 \mathrm{~m}^{2}$ mit einer Leistung bis zu 100 MW.

M. Picon-Nunez, G. T. Polley, E. Torres-Reyes, A. Gallegos-Munoz: „Surface selection and design of plate-fin heat exchangers (Auswahl der Rippenform und Auslegung von berippten Plattenwärmeübertragern) “ [10] In diesem Beitrag gehen die Autoren auf Designkriterien für berippte Plattenwärmeübertrager ein. Sie zeigen, dass die optimale Rippengeometrie dann gefunden ist, wenn bei vorgegebenem Druckverlust der Medien zu einem Wärmeübertrager minimalen Volumens führt. Dazu leiten sie entsprechende Gleichungen her und entwickeln ein „Volume performance index" (VIP) genanntes Konzept, mit dessen Hilfe das Optimum bewertbar ist. Anschließend stellen die Autoren ein thermo-hydraulisches Modell vor, welches die Verbindung zwischen Druckverlust und dem Wärmeübergangskoeffizienten herstellt.

Da berippte Plattenwärmeübertrager sich deutlich von den von uns getesteten Plattenwärmeübertragern unterscheiden, erscheint die Übertragung der angegebenen Beziehungen zweifelhaft. Die generelle Herangehensweise zum Aufdecken des optimalen Designs und die Schaffung eines Kriteriums VIP sollte aber auch im Rahmen unserer Arbeit geprüft werden. 
G. Jonsson, O. P. Palsson: „Use of Empirical Relations in the Parameters of Heat-Exchanger Models (Verwendung empirischer Beziehungen für die Parameter von Wärmeübertrager-Modellen)“ [11]

Die Verfasser zeigen die Verwendung empirischer Beziehungen der Wärmeübergangskoeffizienten in Wärmeübertragermodellen. Mit deren Hilfe ist es möglich, das instationäre Verhalten des Wärmeübertragers über eine große Spannweite von Arbeitsbedingungen abzubilden. Als Parameter des Modells finden NTU-Werte, die Exponenten der Reynoldszahl in den Beziehungen für den Wärmeübergang sowie Zeitkonstanten (Verhältnis der Fluidmasse in einer Zelle zum gesamten Massenstrom) Verwendung. Die Parameter werden direkt durch Minimierung der Differenz zwischen gemessenen Austrittstemperaturen und den aus dem Modell berechneten Werten bestimmt.

Diese Arbeit kann uns Anhaltspunkte für die Auswertung der vorgenommenen Messungen, gegebenenfalls auch für die Auswertung instationärer Messungen geben. Interessant erscheint dies insbesondere unter dem Aspekt, dass die Messungen quasi durchgängig nur im (geringen) Teillastbereich erfolgten.

B. P. Rao, P. K. Kumar, S. K. Das: „Effect of flow distribution to the channels on the thermal performance of a plate heat exchanger (Einfluss der Strömungsverteilung in den Kanälen auf das thermische Verhalten eines Plattenwärmeübertragers)" [12]

Die Autoren beschreiben eine mathematische Methode zur Modellierung des thermischen Verhaltens von Plattenwärmeübertragern. Dabei berücksichtigen sie in ihrem Modell die unterschiedliche Strömungsverteilung in den Kanälen des Wärmeübertragers. So führt ihr Modell für unterschiedliche Anordnung der Ein- und Auslassstutzen („U-Typ“, „Z-Typ“) zu unterschiedlichen Lösungen. Die Effektivität der Wärmeübertrager unterschiedlicher Strömungsführung

$\varepsilon=\frac{\left(\dot{m} c_{p}\right)_{1}\left(T_{1, \text { out }}-T_{1, \text { in }}\right)}{\left(\dot{m} c_{p}\right)_{\min }\left(T_{2, \text { in }}-T_{1, \text { in }}\right)}$

wird in Abhängigkeit von NTU aufgetragen. Die Ergebnisse zeigen, dass für die Auslegung von Apparaten strömungsabhängige Wärmeübertragungskoeffizienten in der Form $\mathrm{Nu}=\mathrm{C}$ Ren Prb benutzt werden sollten. Der Koeffizient n variiert zwischen 0,32 und 0,73 und wird bestimmt vom Strömungsverhalten über den Platten.

Diese Arbeit ist hinsichtlich der Bestimmung der Effektivität des Wärmeübertragers auch für unsere Anwendung interessant und kann direkt auf unsere Messungen übertragen werden.

H. H. Ingwersen: „Auslegungskriterien für Plattenwärmeübertrager" [13]

Der Autor geht in allgemeiner Weise auf die Vorzüge und die Auslegung von Plattenwärmeübertragern ein. Schwerpunkte der Darstellung sind die Anpassung der Prägung an die sogenannte „thermische Länge“ und die bei Plattenwärmeübertragern unkritische „Fouling“-Problematik. Abschließend geht der Autor kurz auf die exergetischen Vorteile von Plattenwärmeübertragern ein, da diese mit sehr geringen Temperaturdifferenzen wirtschaftlich betrie- ben werden können. Der Exergieverluststrom wird bei der Wärmeübertragung mit

$E_{v}=T_{u} \frac{T_{B}-T_{A}}{T_{A} \cdot T_{B}} \dot{Q}$

beschrieben (Tu Umgebungstemperatur, TA und TB Temperatur der Medien).

Für unsere Arbeit hat dieser Beitrag nur informativen Charakter und bietet keine grundsätzlich neuen Erkenntnisse.

J. Eggert: „Turbulenz durch Mehrfeldprägung - Asymmetrische Plattenstruktur für effizient genutzte Wärmeübertragerflächen [14]

Der Autor geht auf den Einsatz der Mehrfeldprägetechnik für Plattenwärmeübertrager ein, die bei deutlich unterschiedlichen Volumenströmen der beiden Medien betrieben werden. Dadurch können die Apparate besser auf die Auslegungsbedingungen angepasst werden und eine Überdimensionierung oder aufwändige Mehrwegigkeit vermieden werden. Er stellt mehrere Plattenformen skizzenhaft vor und geht auf ihre Einsatzgebiete kurz ein.

Für unsere Arbeit hat dieser Beitrag nur informativen Charakter. Wenn es jedoch um den Entwurf neuer Platten für die oben angesprochenen Einsatzbedingungen geht, sollte dieser Artikel den Einstieg für eine vertiefende Recherche bieten.

M. K. Bassiouny: „Experimentelle und theoretische Untersuchungen über Mengenstromverteilung, Druckverlust und Wärmeübergang in Plattenwärmetauschern" [15]

Die Arbeit enthält einen Algorithmus zur iterativen Bestimmung des Wärmeübergangskoeffizienten $\alpha$ aus den Wärmedurchgangskoeffizienten k mit Hilfe der gemessenen Ein- und Austrittstemperaturen und Fluidströme. Da sich bei der Auswertung der experimentellen Arbeiten im Rahmen dieses Vorhabens genau diese Frage stellt, soll hier näher auf Bassiounys Algorithmus eingegangen werden:

Ausgangsgleichung ist die bekannte ähnlichkeitstheoretische Beziehung

$N u=c \cdot \operatorname{Re}^{a} \cdot \operatorname{Pr}^{b}$

Mit den bekannten dimensionslosen Kennzahlen

$N u=\frac{\alpha \cdot d_{h}}{\lambda}(2), \operatorname{Re}=\frac{w \cdot d_{h}}{v}(3)$ und $\operatorname{Pr}=\frac{\eta \cdot c_{p}}{\lambda}$

Ziel ist, für Gleichung (1), die Konstanten a, b und c so zu bestimmen, dass die Differenz zwischen berechnetem und dem gemessenen Wärmedurchgangskoeffizienten, $\mathrm{k}_{\mathrm{ber}}$ bzw. $\mathrm{k}_{\mathrm{exp}}$, so klein wie möglich ist:

$\Delta k=\frac{k_{b e r}-k_{\text {exp }}}{k_{\text {exp }}}=\min$.

Für den Exponenten der Prandtl-Zahl findet man in der einschlägigen Literatur b-Werte, die sich im Bereich von $0,3-0,4$ befinden. Speziell für Plattenwärmeübertrager hat sich $b=1 / 3$ bewährt. Die beiden anderen Konstanten $\mathrm{C}$ und a in Gl. (1) müssen iterativ bestimmt werden: 
Aus Gleichungen (1) und (2) erhält man für die Fluide 1 und 2 nach Umformen:

$\frac{c}{\alpha_{1}}=\frac{d_{h, 1} \cdot \operatorname{Re}^{-a} \cdot \operatorname{Pr}^{-b}}{\lambda_{1}}$ (6) und $\frac{c}{\alpha_{2}}=\frac{d_{h, 2} \cdot \operatorname{Re}^{-a} \cdot \operatorname{Pr}^{-b}}{\lambda_{2}}$.

Mit der Definitionsgleichung des Wärmedurchgangskoeffizienten $\mathrm{k}$

$\frac{1}{k}=\frac{1}{\alpha_{1}}+\frac{1}{\alpha_{2}}+\frac{s_{P}}{\lambda_{P}}$.

erhält man durch Erweiterung mit c und anschließende Umformung:

$c=\frac{\frac{c}{\alpha_{1}}+\frac{c}{\alpha_{2}}}{1-\frac{s_{P}}{\lambda_{P}}}$.

Zuerst wird nun mit den Gleichungen (6), (7) und (9) für einen angenommenen Wert des Exponenten a der Re-Zahl eine Konstante $c_{i}$ für jede Messung i bestimmt. Die Konstanten der einzelnen Messungen werden aufsummiert und durch die Anzahl der Messungen $n$ dividiert:

$c_{m}=\frac{c_{1}+c_{2}+\ldots+c_{n}}{n}$

Mit dem erhaltenen mittleren c-Wert und den folgenden, aus Gl. (6) und (7) erhaltenen Beziehungen wird der Wärmedurchgangskoeffizient $\mathrm{k}_{\mathrm{ber}}$ bestimmt:

$\alpha_{1}=\frac{c_{m} \cdot \lambda_{1}}{d_{h, 1} \cdot \operatorname{Re}_{1}^{-a} \cdot \operatorname{Pr}_{1}^{-b}}$

$\alpha_{2}=\frac{c_{m} \cdot \lambda_{2}}{d_{h, 2} \cdot \operatorname{Re}_{2}^{-a} \cdot \operatorname{Pr}_{2}^{-b}}$

$k_{\text {ber }}=\frac{1}{\frac{1}{\alpha_{1}}+\frac{1}{\alpha_{2}}+\frac{s_{p}}{\lambda_{p}}}$.

Dieser k-Wert, eingesetzt in Gl. (5), wird schließlich optimiert bis zum Finden des passenden Exponenten a der Reynoldszahl.

Dieser in [15] empfohlende Berechnungsalgorithmus hat Berücksichtigung auch in der neuesten Fassung des VDI-Wärmeatlas [2] zur Berechnung des Druckverlustes und des Wärmeübergangs in Plattenwärmeübertragern mit rechteckigen Platten gefunden. Inwiefern die Gleichungen auch für Platten mit einem Kreisquerschnitt gelten, wie die in diesem Projekt vorgesehenen Apparate, wird nicht beschrieben. Einige der empfohlenen Beziehungen müssen deshalb im Rahmen dieser Arbeit im Hinblick auf ihre Verwendbarkeit für Platten mit Kreisquerschnitt überprüft und ggfs. modifiziert werden.

G. Diaz, M. Sen, T. Schutt, J. Schmadl: „Modellierung des Wärmeübergangs beim Blasensieden in freier Konvektion mit künstlichen neuronalen Netzen. Teil I: Literaturübersicht, Teil II: Einstoffsysteme“ [18][19]

Eine völlig andere, unkonventionelle Möglichkeit zur Modellierung des Wärmeübergangs kündigt sich für die Zukunft an durch die Anwendung neuronaler Netzwerke: Dazu gibt es bislang nur wenige aber sehr erfolgversprechende Korrelationsversuche mit dem ANN-Typ Multilayerperceptron
(MLP) [16, 17]. Da konventionelle Gleichungen generell weniger aufwendig zu finden und einfacher zu handhaben sind, wird die Auswertung der eigenen Messungen zunächst konventionell versucht, MLP-Modelle kommen aber in Frage, falls mit konventionellen Rechenansätzen keine befriedigenden Ergebnisse erzielbar sind.

\section{Verwendbarkeit von Literaturangaben in dieser Arbeit}

In dieser Arbeit wird ein Teststand für experimentelle Druckverlust- und Leistungsmessungen an Plattenwärmeübertragern verwendet, der an anderer Stelle beschrieben wird. Dabei werden je zwei Ein- und Austrittstemperaturen sowie die Druckverluste und Volumenströme der beiden Fluide gemessen. Die Messtechnik ist Gegenstand einer getrennten Betrachtung.

a) Auswertung der Druckverlustmessungen:

Als erster Schritt kann dazu kann die Definitionsgleichung des Druckverlustbeiwertes $\xi$ verwendet werden:

$\xi=\frac{2 \cdot \Delta p \cdot d_{h}}{\rho \cdot w^{2} \cdot L_{P}}$

mit dem Reibungsdruckabfall $\Delta$ p über die Länge der Platte und dem hydraulischen Durchmesser dh, definiert als:

$d_{h}=\frac{4 \cdot \hat{a}}{\Phi}$.

Dabei ist $\hat{a}$ die Amplitude des sinusförmigen Wellenmusters und $\Phi$ das Verhältnis der gewellten zur projizierten Plattenfläche, auch Flächenvergrößerungsfaktor genannt, wie in Abb. 3 dargestellt. Der Flächenvergrößerungsfaktor $\Phi$ ist seinerseits abhängig von dem Verhältnis der Amplitude zur Wellenlänge, oder einfacher von der Wellenzahl:

$X=\frac{2 \cdot \pi \cdot \hat{a}}{\Lambda}$

(siehe auch Abbildung 3). Für die sinusförmige Wellung kann er näherungsweise nach der Formel

$\Phi(X) \approx \frac{1}{6}\left(1+\sqrt{1+X^{2}}+4 \cdot \sqrt{1+\frac{X^{2}}{2}}\right)$

berechnet werden.

In die Re-Zahl wir der hydraulische Durchmesser nach (15) und eine Strömungsgeschwindigkeit w eingesetzt, welche sich berechnet aus dem Volumenstrom durch den Spalt zwischen zwei Platten, dividiert durch die mittlere Querschnittsfläche des Spaltes:

$w=\frac{\dot{V}_{\text {Spalt }}}{2 \cdot \hat{a} \cdot B_{P}}$.

b) Auswertung der Wärmeübergangsmessungen: Ausgangsgleichung ist der 1. Hauptsatz der Thermodynamik in einer für die zu untersuchenden, als adiabaten Kontrollraum betrachteten Testapparate passenden Form:

$\sum \dot{H}_{a u s, i}-\sum \dot{H}_{e i n, i}=\Delta \dot{H}_{w}+\Delta \dot{H}_{\ddot{O} l}=0$

mit i = Wasser $(\mathrm{W})$, Öl 
Für Flüssigkeiten gilt: $\Delta \dot{H}_{i}=\dot{M}_{i} c \Delta T+\dot{V}_{i} \Delta p_{i}$

Mit (19) und (20) ergibt sich:

$\dot{M}_{W} c_{W} \Delta T_{W}+\dot{V}_{W} \Delta p_{W}+\dot{M}_{\ddot{O} l} c_{\ddot{O} l} \Delta T_{\ddot{O} l}+\dot{V}_{\ddot{O} l} \Delta p_{\ddot{O} l}=0$

Ausgehend von dieser Energiebilanz lassen sich die Wärmedurchgangskoeffizienten $\mathrm{k}\left[\mathrm{W} /\left(\mathrm{m}^{2} \mathrm{~K}\right)\right]$ berechnen nach der bekannten Gleichung:

$k=\frac{\dot{Q}}{A \cdot \Delta T_{\ln }}$

mit dem Wärmestrom

$\dot{Q}=\dot{M}_{W} c_{W} \Delta T_{W}+\dot{V}_{W} \Delta p_{W}=-\left(\dot{M}_{\ddot{O l l}} c_{\ddot{O} l} \Delta T_{\ddot{O} l}+\dot{V}_{\ddot{O} l} \Delta p_{\ddot{O} l}\right)$,

A als Wärmeübertragungsfläche und $\Delta T_{\mathrm{ln}}$ als integrales (logarithmisches)Temperaturgefälle.

A ist bekannt, $\dot{Q}$ und $\Delta T_{\ln }$ werden experimentell ermittelt und daraus der k-Wert berechnet. Für die weitere wärmetechnische Auswertung wird die beschriebene Methode von Bassiouny [] verwendet um die $\boldsymbol{\alpha}$-Werte zu berechnen. Für die weitere Auswertung werden u.a. empirische Gleichungen vom Typ (1) aufgestellt.

Wegen der periodischen Struktur der Strömungskanäle kann man den Wärmeübergang mit brauchbarer Näherung aus der von Lévêque angegebenen asymptotischen Lösung abschätzen:

$N u=1,615\left[\left(\frac{\xi \cdot \operatorname{Re}}{64}\right) \cdot \operatorname{Re} \cdot \operatorname{Pr} \cdot \frac{d_{h}}{L}\right]^{1 / 3}$.

Bei ausgebildeter laminarer Rohrströmung ist darin nach dem Hagen-Poiseuille-Gesetz $\left(\frac{\xi \cdot \operatorname{Re}}{64}=1\right)$. Umgeformt geschrieben gilt:

$N u=0,4038 \cdot\left(\xi \cdot \operatorname{Pr} \cdot \frac{d_{h}}{L} \cdot \operatorname{Re}^{2}\right)^{1 / 3}$.

Für die Länge L muss dabei der Abstand zwischen zwei Kreuzungspunkten eingesetzt werden:

$\frac{d_{h}}{L}=\left(\frac{d_{h}}{\Lambda}\right) \cdot \sin (2 \varphi)$.

$\varphi$ ist der in der Abb.3 dargestellte Winkel der WellenPrägung.

Ein detaillierter Vergleich mit weiteren, bisher publizierten Daten zeigt, dass die Abhängigkeit der Nu-Zahl von der Re-Zahl, insbesondere bei technischen Plattenwärmeübertragern etwas besser wiedergegeben werden kann, wenn man die Lévêque-Gleichung geringfügig modifiziert. Der Exponent 1/3 wird durch wird durch den etwas größeren Wert $\mathrm{q}=0,374$ ersetzt. Der geometrische Parameter

$\left(\frac{d_{h}}{\Lambda}\right)$

wird zweckmäßigerweise in den dann ebenfalls empirisch modifizierten Vorfaktor $(c=0,122)$ mit einbezogen, da die Mehrzahl der Hersteller die geometrischen Details ihrer Prägemuster nicht publiziert:

$N u=c \cdot \operatorname{Pr}^{1 / 3} \cdot\left(\frac{\eta}{\eta_{w}}\right)^{1 / 6} \cdot\left(\xi \cdot \operatorname{Re}^{2} \cdot \sin (2 \varphi)\right)^{q}$

\section{Der Korrekturfaktor}

$\left(\frac{\eta}{\eta_{w}}\right)^{1 / 6}$

berücksichtigt die Temperaturabhängigkeit der Viskosität.

Diese Gleichungen können nur als Ausgangsbasis betrachtet werden, da sie für rechteckige Platten entwickelt worden sind. Die in diesem Vorhaben zu untersuchende kreisrunde Plattenform ist bislang weder experimentell untersucht noch theoretisch beschrieben worden. Sollte sich herausstellen, dass Gleichungen vom Typ (1) prinzipiell anwendbar ist, werden für die in dieser Arbeit zu untersuchenden Platten möglicherweise andere Exponenten C und a in Gl. (1) gefunden werden müssen.

\section{Literatur}

[1] VDI-Wärmeatlas, 9. Auflage, Springer-Verlag, 2002, Kapitel $\mathrm{Mm}$.

[2] Z. H. Ayub: "Plate Heat Exchanger Literature Survey and new Heat Transfer and pressure drop correlations for Refrigerant Evaporators", Heat Transfer Engineering (2003), 24, 3-16.

[3] M. Gaiser: „Strömungs- und Transportvorgänge in gewellten Strukturen“, Dissertation Universität Stuttgart, 1990, 195 pp.

[4] R. Hoffmann, W. Krug und M. Arras: „Auswertung experimenteller Untersuchungen des Wärmeübertragungsprozesses im Plattenwärmeübertrager", Luft- und Kältetechnik 1985, 3, 160-162.

[5] W. W. Focke, J. Zachariades und I. Olivier: "The effect of the corrugation inclination angle on the thermohydraulic performance of plate heat exchangers", Heat Mass Transfer (1985) 28(8), 1469-1479.

[6] M. Ciofalo, I. Di Piazza, J. A. Stasiek: "Investigation of flow and heat transfer in corrugated-undulated plate heat exchangers", Heat and Mass Transfer (2000) 36(5), 449-462.

[7] P. Loose: „Teillastverhalten von Plattenwärmeübertragern“, Euroheat .amp. Power - Fernwärme International 29(4), 5257.

[8] Ranganayakulu, Ch., K. N. Seetharamu: „The combined effects of inlet fluid flow and temperature nonuniformity in cross flow plate-fin compact heat exchanger using finite element method“, Heat Mass Transfer (1997) 32(5), 375383.

[9] Klein, A. : „Wärmeübertrager“, Chemie Ingenieur Technik (2000) 72(12), 1495-1499.

[10] M. Picon-Nunez, G. T. Polley, E. Torres-Reyes, A. GallegosMunoz: „Surface selection and design of plate-fin heat“, Appl. Thermal Eng 19(1999), 917-931.

[11] G. Jonsson, O. P. Palsson: „Use of Empirical Relations in the Parameters of Heat-Exchanger Models", Ind. Eng. Chem. Res. (1991) 30(6), 1193-1199.

[12] B. P. Rao, P. K. Kumar, S. K. Das: „Effect of flow distribution to the channels on the thermal performance of a plate heat", Chemical Engineering and Processing (2002) 41, 49-58.

[13] H. H. Ingwersen: „Auslegungskriterien für Plattenwärmeübertrager", Chem.-Tech. (Heidelberg) (1997), 26(8), 13-14. 
[14] J. Eggert: „Turbulenz durch Mehrfeldprägung - Asymmetrische Plattenstruktur für effizient genutzte Wärmeübertragerflächen", Chemie Technik (Heidelberg, Germany) (2001) 30(6), 20-22.

[15] M. K. Bassiouny: „Experimentelle und theoretische Untersuchungen über Mengenstromverteilung, Druckverlust und Wärmeübergang in Plattenwärmetauschern“, Dissertation Universität Karlsruhe (1985) in Fortschr.-Ber. VDI Reihe 6 Nr.181, Düsseldorf, VDI-Verlag 1985, 308 S..

[16] A. Pacheca-Vega, M. Sen, K. T. Yang, R. L. McClain: „Genetic-algorithm-based predictions of fin-tube heat exchanger performance“, Int. Heat Transfer Conf., Korea, 1998.

[17] G. Diaz, M. Sen, K. T. Yang, R. L. McClain: „Simulation of heat exchanger performance by artificial neural networks", under review for Int. J. of HVAC\&R Research (ASHRAE).

[18] G. Diaz, M. Sen, T. Schutt, J. Schmadl: „Modellierung des Wärmeübergangs beim Blasensieden in freier Konvektion mit künstlichen neuronalen Netzen. Teil I: Literaturübersicht", Wissenschaftliche Beiträge TFH Wildau, Heft 2/2000, S3035 .

[19] G. Diaz, M. Sen, T. Schutt, J. Schmadl: „Modellierung des Wärmeübergangs beim Blasensieden in freier Konvektion mit künstlichen neuronalen Netzen. Teil II: Einstoffsysteme", Wissenschaftliche Beiträge TFH Wildau, Heft 2/2000, S. 3641.

\section{Autoren}

Prof. Dr.-Ing. Josef Schmadl

Technische Fachhochschule Wildau

Fachbereich Ingenieurwesen/Wirtschaftsingenieurwesen

Technikum für Thermische Verfahrenstechnik

Tel. +493375 508-110

jschmadl@igw.tfh-wildau.de

Dipl.-Ing. Jens-Uwe Gerking

Technische Fachhochschule Wildau

Fachbereich Ingenieurwesen/Wirtschaftsingenieurwesen Technikum für Thermische Verfahrenstechnik

Tel. +49 3375 508-177

gerking@vt.tfh-wildau.de

Dipl.-Ing. (FH) Maik Haß

Dr. Ingrid Schult

Dipl.-Ing. Joachim Schult

Caloperm $\mathrm{GmbH}$

Am Möllenberg 40, 15751 Niederlehme

Tel. +49 3375 5185-18 